\title{
Valores de referência para plumbemia em uma população urbana do Sul do Brasil
}

\author{
Monica M. B. Paoliello, ${ }^{1}$ Paulo R. Gutierrez, ${ }^{2}$ Conceição A. Turini, ${ }^{1}$ \\ Tiemi Matsuo, ${ }^{3}$ Leda Mezzaroba, ${ }^{1}$ Décio S. Barbosa, ${ }^{1}$ \\ Sandra R. Q. Carvalho, ${ }^{1}$ Aparecida L. P. Alvarenga, ${ }^{1}$ \\ Maria Inês Rezende, ${ }^{1}$ Garcia A. Figueiroa, ${ }^{4}$ Viviane G. M. Leite, ${ }^{5}$ \\ Amanda C. Gutierrez, ${ }^{6}$ Bruno C. R. Lobo $^{7}$ e Reginaldo A. Cascales ${ }^{7}$
}

RESUMO Objetivo. Descrever os valores de referência para chumbo em sangue em uma população urbana do Município de Londrina, Estado do Paraná, Brasil.

Métodos. A população de referência foi constituída por 520 voluntários adultos avaliados de novembro de 1994 a dezembro de 1996. Os critérios de exclusão foram: exposição ocupacional ao chumbo, exposição através de hábitos, fumar mais de 10 cigarros por dia e morar perto de indústrias ou de locais que utilizam o chumbo em seus processos produtivos. Também foram excluídos indivíduos com valores de exames clínicos e laboratoriais fora da normalidade, doenças crônicas e distúrbios cardiovasculares. Os teores de chumbo em sangue foram determinados por espectrofotometria de absorção atômica com chama de ar-acetileno. O limite de detecção obtido foi 1,23 $\mu \mathrm{g} / \mathrm{dL}$. Após as análises de chumbo em sangue, foram determinados valor mínimo, primeiro quartil, mediana, terceiro quartil e valor máximo; média geométrica; intervalo de confiança de 95\%; intervalo experimental; e valor de referência.

Resultados. Os valores de referência para plumbemia variaram de 1,20 a 13,72 $\mu \mathrm{g} / \mathrm{dL}$. A média geométrica foi igual a 5,5 $\mu \mathrm{g} / \mathrm{dL}$.

Conclusões. Em geral, os valores obtidos no presente estudo são mais baixos do que aqueles obtidos em outros países. Estudos adicionais deveriam enfocar a obtenção de valores de referência em outras populações brasileiras que vivem em regiões mais industrializadas.

Palavras chave Chumbo em sangue, valores de referência, população de referência.

Com o aumento da industrialização nos últimos 40 anos no Brasil, especialmente com a chegada da indústria automobilística, a exposição ao chumbo tornou-se um problema de saúde pública. Além da interferência na síntese da heme, o chumbo causa uma variedade de efeitos adversos à saúde, incluindo danos ao sistema nervoso central e periférico, doenças renais e anormalidades reprodutivas. Sua vasta utilização em processos produti- vos expõe não somente os indivíduos que trabalham com este metal, mas também a população em geral. $\mathrm{O}$ estabelecimento de valores de referência para chumbo representa o ponto de partida para que a monitorização bio-

\footnotetext{
Universidade Estadual de Londrina, Centro de Ciências da Saúde, Departamento de Patologia Aplicada, Legislação e Deontologia, Londrina, PR, Brasil. Correspondência e pedidos de separatas devem ser enviados a Monica Maria Bastos Paoliello no seguinte endereço: Hospital Universitário Regional do Norte do Paraná, Departamento de Patologia Aplicada, Legislação e Deontologia,
}

Avenida Roberto Koch 60, CEP 86038-440, Londrina, PR, Brasil. Tel: +55-43-371-2200/324-2491; email: monibas@onda.com.br

2 Universidade Estadual de Londrina, Centro de Ciências da Saúde, Departamento Materno-Infantil e Saúde Comunitária.

3 Universidade Estadual de Londrina, Centro de Ciências da Saúde, Núcleo de Epidemiologia Clínica.
4 Prefeitura do Município de Londrina.

5 Universidade Estadual de Londrina, Faculdade de Farmácia e Bioquímica.

6 Universidade Estadual de Londrina, Faculdade de Química.

7 Universidade Estadual de Londrina, Hospital Universitário Regional do Norte do Paraná. 
lógica se torne uma prática rotineira no âmbito da vigilância à saúde e nos estudos epidemiológicos.

Os valores de referência são definidos a partir da determinação da quantidade de um dado elemento em um indivíduo ou população. Tais indivíduos ou populações são selecionados segundo critérios predeterminados, levando-se em consideração seu estado de saúde. Para o estabelecimento de valores de referência adequados é necessário controlar os diversos fatores ligados à variabilidade biológica (características da população em estudo) e analítica (coleta, tratamento e análise das amostras biológicas e análise dos dados). A observação incorreta desses fatores implica em variabilidade muito ampla, sobretudo na dosagem de elementos presentes nos líquidos biológicos em concentrações muito baixas, na ordem de traços, como é o caso do chumbo (1).

No Brasil, em função da falta de estudos para estabelecer valores de referência para a exposição ao chumbo, é comum a utilização de dados de outros países, muitas vezes inadequados para a nossa realidade $(2,3)$. Assim, o objetivo do presente estudo foi descrever os valores de referência para chumbo em sangue em uma população urbana do Município de Londrina, Estado do Paraná, Brasil.

\section{MATERIAIS E MÉTODOS}

\section{Definição da população de referência}

Foi realizada uma seleção $a$ posteriori a partir da população geral. Participaram do estudo voluntários das diversas regiões da área urbana do Município de Londrina, contatados através de associações de moradores, entidades religiosas, conselhos regionais de saúde e instituições públicas. O Município de Londrina apresenta uma população total estimada em 446849 habitantes (4) e sua economia baseia-se na produção de serviços, em especial nas áreas de saúde e comércio. A cidade é pouco industrializada, pos- suindo como possíveis fontes de exposição ao chumbo duas fábricas de baterias de médio porte e várias retificadoras. A coleta das amostras e demais procedimentos foram realizados nos finais de semana em diversos locais do Município (salas de aula, salões paroquiais e, com menor freqüência, centros de saúde), no período de novembro de 1994 a dezembro de 1996.

Foram utilizados os seguintes critérios de exclusão: exposição ocupacional ao chumbo, exposição através de hábitos (por exemplo, pescarias freqüentes, onde comumente se coloca o "chumbinho" fixado à linha de pesca na boca, artesanato com cerâmicas vitrificadas), fumar mais de 10 cigarros por dia e morar perto (menos de $3 \mathrm{~km}$ ) de indústrias ou de outros locais que utilizam o chumbo em seus processos produtivos (5-7). Para averiguar essas condições de exposição, foi aplicado um questionário.

Foram selecionados indivíduos considerados sadios. Para isso, foram realizados anamnese e exame físico, além dos seguintes exames laboratoriais: hemograma, glicose, uréia, creatinina, gama-glutamiltransferase, alanina-aminotransferase, aspartatoaminotransferase, colesterol total, triglicerídeos e exame parcial de urina. Foram excluídos sujeitos que apresentaram valores clínicos e laboratoriais fora da faixa de normalidade, além daqueles afetados por doenças crônicas como alcoolismo e distúrbios cardiovasculares $(4,7)$.

\section{Dimensionamento da amostra}

A amostra foi dimensionada levando-se em consideração o número de habitantes da população adulta da região urbana do Município de Londrina (4), a média de plumbemia $(8,28 \mu \mathrm{g} / \mathrm{dL})$ e o desvio padrão $(4,17 \mu \mathrm{g} / \mathrm{dL})$ obtidos em estudos anteriores realizados no Município (3). Com base nessas estimativas, a amostra foi calculada em um mínimo de 400 indivíduos, com nível de confiança de 95\% e 5\% de erro na estimativa. Inicialmente, foram selecionados 739 vo- luntários. Após a exclusão dos que não atendiam aos critérios preestabelecidos, foram selecionados 538 sujeitos para participação no estudo.

\section{Técnica analítica e controle de qualidade}

Os teores de chumbo em sangue foram determinados por espectrofotometria de absorção atômica com chama de ar-acetileno, utilizando-se um equipamento Perkin Elmer modelo 3110. O método utilizado se baseia na complexação do chumbo com pirrolidinaditiocarbamato de amônio e extração com metilisobutilcetona (8). O sangue sofreu uma pré-concentração em quatro vezes. O limite de detecção obtido foi $1,23 \mu \mathrm{g} / \mathrm{dL}$.

Os valores de recuperação encontrados para a curva padrão $(5,10,20,40$, 60, 80 e $100 \mu \mathrm{g} / \mathrm{dL})$ foram em média $101 \%$.

Verificou-se a variabilidade analítica através da adoção de controles internos de qualidade, com coeficientes de variação inferiores a 5\% nos testes de reprodutibilidade inter e intra-ensaio.

A garantia da qualidade nas determinações de plumbemia foi monitorada por um programa externo de controle de qualidade, com 16 determinações anuais, coordenado pelo Instituto Adolfo Lutz (setor de Equipamentos Especializados) em São Paulo, Brasil.

\section{Análise estatística}

Após a realização das análises de chumbo em sangue, foram determinados:

a) valor mínimo, primeiro quartil, mediana, terceiro quartil e valor máximo;

b) média geométrica;

c) intervalo de confiança de 95\% (IC95\%), obtido através do intervalo $\overline{\mathrm{x}} \pm \mathrm{z}_{\alpha / 2} \mathrm{~S}(\overline{\mathrm{x}})$ onde $\overline{\mathrm{x}}$ é o valor médio, $z_{\alpha} / 2$ é o valor da distribuição normal, considerando que a amostra do estudo é grande $(n>30)$ e $\mathrm{s}(\overline{\mathrm{x}})$ é o erro padrão da média; 
d) intervalo experimental, delimitado pelo menor e maior valor observado;

e) valor de referência, obtido através do intervalo $\bar{x} \pm 2$ s, onde $\bar{x}$ é o valor médio e s é o desvio padrão dos valores observados. Se o menor valor observado estiver abaixo de $\bar{x}-2$, o intervalo será delimitado pelo menor valor observado e $\bar{x}+2$ s.

f) intervalo de incerteza, delimitado por $\bar{x}+2$ s e pelo maior valor observado.

A comparação entre os valores de plumbemia para o sexo feminino e masculino foi analisada pelo teste $t$ de Student. O nível de significância adotado para o teste foi de $5 \%$.

\section{RESULTADOS}

No presente estudo, dos 538 sujeitos analisados, 18 foram excluídos para fins de análise estatística, pois apresentaram valores de plumbemia abaixo do limite de detecção (apesar de alguns autores considerarem que é possível se utilizar valores a partir da metade do limite mínimo detectável) $(9,10)$. Assim, os níveis de plumbemia de 520 indivíduos foram analisados. Destes, 301 eram do sexo feminino e 219 do sexo masculino, com idade média igual a 40 anos. A tabela 1 apresenta os resultados das análises de chumbo em sangue segundo o sexo.

A figura 1 apresenta a distribuição dos valores para chumbo em sangue segundo quartis, mostrando um ponto extremo (correspondente a 23,9 $\mu \mathrm{g}$ / dL). Minoia et al. (7) afirmam que as condições que levam sujeitos a apresentar valores dentro do intervalo de incerteza, que é o intervalo dos altos valores observados, devem ser precisamente determinadas. Assim, para verificar se esse ponto extremo deveria permanecer no conjunto dos dados, efetuou-se uma análise detalhada desse caso, bem como daqueles sujeitos que apresentaram níveis de chumbo mais altos. Após a realização da nova análise, observou-se que os dados clínicos, laboratoriais e ocupa-

TABELA 1. Valores de chumbo em sangue $(\mu \mathrm{g} / \mathrm{dL})$ em adultos sadios do Município de Londrina (PR), Brasil, 1994 a 1996

\begin{tabular}{lccc}
\hline & \multicolumn{2}{c}{ Sexo } & \\
\cline { 2 - 3 } \multicolumn{1}{c}{ Estatística } & $\begin{array}{c}\text { Feminino } \\
(n=301)\end{array}$ & $\begin{array}{c}\text { Masculino } \\
(n=219)\end{array}$ & $\begin{array}{c}\text { Total } \\
(n=520)\end{array}$ \\
\hline Valor mínimo & 1,2 & 1,2 & 1,2 \\
Primeiro quartil & 3,6 & 3,5 & 3,6 \\
Mediana & 5,7 & 5,6 & 5,7 \\
Terceiro quartil & 8,1 & 9,0 & 8,3 \\
Valor máximo & 23,0 & 23,0 & 23,0 \\
Média geométrica & 5,4 & 5,6 & 5,5 \\
Intervalo de confiança de 95\% & $6,46 \pm 0,40$ & $6,62 \pm 0,49$ & $6,52 \pm 0,30$ \\
Intervalo experimental & $1,20-23,00$ & $1,20-23,0$ & $1,20-23,00$ \\
Valor de referência & $1,20-13,49$ & $1,20-14,04$ & $1,20-13,72$ \\
Intervalo de incerteza & $13,49-23,00$ & $14,04-23,00$ & $13,72-23,00$ \\
\hline
\end{tabular}

cionais dos mesmos se enquadraram nos critérios preestabelecidos, optando-se pela permanência desses valores no conjunto dos dados.

A diferença entre o sexo feminino e o masculino em relação ao valor médio de plumbemia não foi significativa $(P<0.05$, teste $\mathrm{t}$ de Student $)$.

\section{DISCUSSÃO}

Os principais resultados obtidos neste estudo - ou seja, valores de referência entre 1,20 e 13,72 $\mu \mathrm{g} / \mathrm{dL}$, mediana igual a $5,7 \mu \mathrm{g} / \mathrm{dL}$ e média geométrica igual a $5,5 \mu \mathrm{g} / \mathrm{dL}$ (tabela 1 ) de um modo geral encontraram-se abaixo daqueles relatados em estudos realizados em outros países utilizando metodologia semelhante. Na Itália, Morisi et al. (11) obtiveram mediana para plumbemia igual a $15,30 \mu \mathrm{g} / \mathrm{dL}$ para homens e $10 \mathrm{mg} / \mathrm{dL}$ para mulheres. Minoia et al. (7), também na Itália, obtiveram média igual a 15,77 \pm 9,9 $\mu \mathrm{g} / \mathrm{dL}$ e valores de referência entre 3,97 e 27,57 $\mu \mathrm{g} / \mathrm{dL}$. Ainda naquele país, Apostoli e Alessio (12) observaram média de 13,80 \pm 8,0 $\mu \mathrm{g} / \mathrm{dL}$. Numa compilação de dados obtidos em 55 países, Iyengar e Wolttiez (5) constataram valor de mediana igual a 12,30 $\mu \mathrm{g} / \mathrm{dL}$.

Resultados semelhantes aos obtidos no presente estudo foram observados em uma comparação entre populações urbanas da China e Japão por Zhang et al. (13), que encontraram médias geométricas para plumbemia de 5,67 $\mu \mathrm{g} / \mathrm{dL}$ e 3,21 $\mu \mathrm{g} / \mathrm{dL}$ em mulheres chinesas e japonesas, respectivamente. Além disso, Montesanti et al. (14) observaram, na população de uma cidade italiana, valores médios de plumbemia iguais a 7,04 $\pm 3,69 \mu \mathrm{g} / \mathrm{dL}$ e mediana igual a 6,0 $\mu \mathrm{g} / \mathrm{dL}$. Entretanto, aquele estudo também incluiu crianças.

A comparação com os poucos estudos realizados sobre o tema no Brasil, que constataram níveis de plumbemia mais elevados em cidades brasileiras industrializadas (15-17), foi dificultada pelas diferenças metodológicas. Alguns autores confirmaram a associação entre variáveis como sexo e idade e nível de chumbo no sangue $(11,18)$. Entretanto, no presente estudo, não se observou diferença estatisticamente significativa entre os níveis de plumbemia segundo o sexo. Schuhmacher et al. (19), analisando os níveis de plumbemia de 488 moradores de uma província espanhola, também não observaram diferenças entre os sexos. O mesmo ocorreu na população estudada por Montesanti et al. (14).

Os resultados obtidos parecem ser compatíveis com as características do Município de Londrina, que apresenta pouca industrialização, destacando-se mais na área de prestação de serviços. 
FIGURA 1. Níveis de plumbemia no Município de Londrina (PR), Brasil, 1994 a 1996

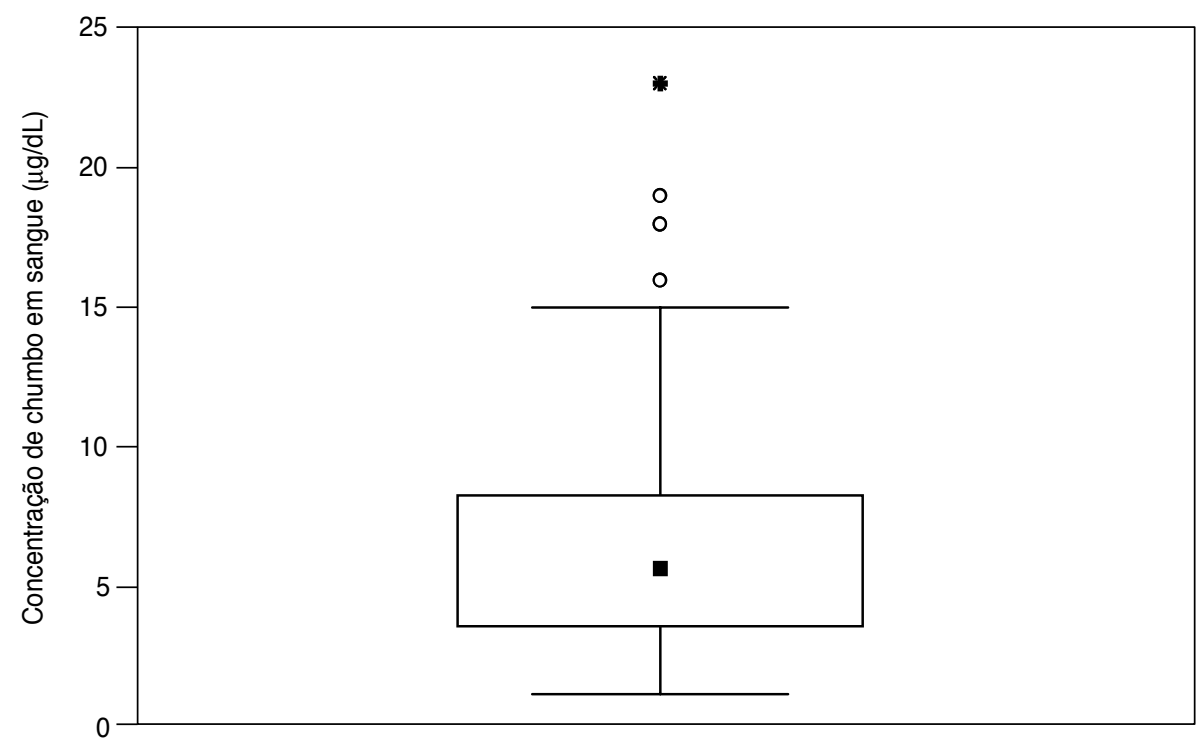

Isso reforça a idéia de que níveis mais altos de plumbemia são encontrados em populações residentes em regiões industrializadas, como observaram Gerhardsson et al. (20), que examina- ram mais de 1000 trabalhos publicados entre 1980 e 1994 na tentativa de estabelecer valores de referência em populações adultas não expostas ocupacionalmente. Entretanto, mesmo em regiões industrializadas, ou naquelas caracterizadas pela existência de indústrias que usam o chumbo em seus processos produtivos, alguns autores verificaram uma tendência na redução da plumbemia na população não exposta ocupacionalmente ao metal (21-23).

Os resultados obtidos no presente estudo podem contribuir para uma adequada biomonitorização de trabalhadores expostos ao chumbo, bem como para a adoção de intervenções preventivas que possam garantir as condições de trabalho e a qualidade de vida da população de Londrina. Entretanto, seria também importante a obtenção de valores de referência em outras populações brasileiras que vivem em regiões mais industrializadas.

Agradecimentos. Os autores agradecem à Fundação Kellogg (Projeto UNI - Londrina, desenvolvido no Centro de Ciências da Saúde da Universidade Estadual de Londrina, Brasil) que financiou parcialmente este trabalho.

\section{REFERÊNCIAS}

1. Apostoli P. Reference values for xenobiotics in biological matrixes: the state of art. G Ital Med Lav 1994;16(1-6):19-24.

2. Salgado PET. Valores de referência para metais. Rev Bras Toxicol 1990;3:35.

3. Paoliello MMB, Gutierrez PR, Turini CA, Matsuo T, Mezzaroba L, Barbosa DS, et al. Valores de referência para plumbemia em população urbana. Rev Saude Publ 1997;31(2):144-8.

4. Fundação Instituto Brasileiro de Geografia e Estatística. Décimo recenseamento geral do Brasil: boletim resumo do município de Londrina. Rio de Janeiro: IBGE; 1991

5. Iyengar V, Wolttiez J. Trace elements in human clinical specimens: evaluation of literature data to identify reference values. Clin Chem 1988;34(3):474-81.

6. Minoia C, Pozzoli L, Cavalleri A, Capodaglio E. Definizione dei valore di riferimento di 30 elementi in trace nei liquid biologici. Em: Societá Italiana di Medicina del Lavoro e Igiene Industriale, ed. $48^{\circ}$ Congresso Nazionale; 1985 set 18-21; Pavia, Italia. Bologna: Monduzzi; 1985. pp. 317-23.

7. Minoia C, Sabbion E, Apostoli P, Pietra R, Pozzoli L, Gallorini M, et al. Trace element reference values in tissues from habitants of the European Community: a study of 46 ele- ments in urine, blood and serum of Italian subjects. Sci Total Environ (Netherlands) 1990;95:89-105.

8. Mitchell DG, Ryan RJ, Aldous KM. The precise determination of lead in whole blood by solvent extraction atomic spectrometry. Atomic Abs News 1972;11:120-1.

9. Brugnone F, Perbellini 1, Giuliari C, Cerpelloni M, Soave M. Blood and urine concentration of chemical pollutants in the general population. Med Lav 1994;85(5):370-89.

10. Wang G, Maranelli G, Perbellini L, Guglielmi $G$, Brugnone F. Reference values for blood toluene in the occupationally non-exposed general population. Int Arch Occup Environ Health 1993; 65(3):201-3.

11. Morisi G, Patriarca M, Carrieri MP, Fondi G, Taggi F. Lead exposure: assessment of the risk for the general Italian population. Ann Ist Super Sanita 1989;25(3):423-35.

12. Apostoli P, Alessio L. Valori di referimento e controllo dei fattori di variabilitá. Em: La Promazione della qualitá dei dati nel monitoraggio biologico. Bologna, Itália: Moderna; 1990. pp. 111-27.

13. Zhang ZW, Moon CS, Watanabe T, Shimbo S, $\mathrm{He} F S, W u Y Q$, et al. Background exposure of urban populations to lead and cadmium: comparison between China and Japan. Int Arch Occup Health 1997; 69(4):273-81.

14. Montesanti M, Morgantini F, Landucci $C$, Rossi L, Biagi C, Castelli S. Livelli ematici di piombo nei bambini della Piana di Lucca. Rilevazione del 1993. Minerva Pediatr 1995; 47(4):119-25.

15. Nogueira DP, Colacioppo S, Souza JMP, Pezza CB, Souza MLA, Gomes JR. Taxa de chumbo em amostra de voluntários "não expostos" habitantes na grande São Paulo. Rev Saude Publ 1979;13(2):147-50.

16. Fernícola NAGG, Azevedo FA. Níveis de chumbo e atividade da desidratase do ácido $\delta$-aminolevulínico ( $\delta$-ALA D) no sangue da população da grande São Paulo, Brasil. Rev Saude Publ 1981; 15(3):272-82.

17. Santos Filho E, Silva RS, Barretto HHC, Inomata ONK, Lemes VRR, Sakuma AM, et al. Concentrações sangüíneas de metais pesados e praguicidas organoclorados em crianças de 1 a 10 anos. Rev Saude Publ 1993;27(1):59-67.

18. Roggi C, Minoia C, Silva S, Ronchi A, Gatti A, Maccarini L. Distribution of blood lead level in a general population. Ann Ig 1995;7(5): 359-67.

19. Schuhmacher M, Domingo JL, Llobet JM. Lead concentration and delta-aminolevulinic acid dehydratase activity in the blood of 
general population of Tarragona Province, Spain. Sci Total Environ 1992;116(3):253-9.

20. Gerhardsson L, Kazantzis G, Schutz A. Evaluation of selected publications on reference values for lead in blood. Scand J Work Environ Health 1996;22(5):325-31.

21. Watanabe T, Nakatsuka H, Shimbo S, Iwami O, Imai Y, Moon CS et al. Reduced cadmium and lead burden in Japan in the past 10 years.
Int Arch Occup Environ Health 1996;68(5): 305-14.

22. Li Donni V, Bagnoli P, Bartoli D, Bavazzano P, Ieri Cavalli $\mathrm{P}$, Landucci $\mathrm{C}$, et al. Blood lead levels in a non-professionally-exposed population from six Tuscan provinces. Ann Ist Super Sanita 1998;34(1):75-80.

23. Sanna E, Cosseddu GG, Floris G, Liguori A, Peretti M, Carbini l. Comparison of blood lead levels in three groups of Sardinian children. Anthropol Anz 1999;57(2):111-21.

Manuscrito recebido em 17 de março de 2000. Aceito em versão revisada em 23 de abril de 2001.

ABSTRACT Objective. To describe the reference values for lead in blood in an urban population in the city of Londrina, in the state of Paraná, Brazil.

Materials and methods. The reference population was composed of 520 adult vo-

Reference values for lead in blood in an urban population in southern Brazil lunteers who were assessed from November 1994 to December 1996. Exclusion criteria were: occupational exposure to lead, exposure through personal habits or practices, smoking more than 10 cigarettes per day, and living near industrial plants or other places that use lead in their production processes. Also excluded were individuals with abnormal clinical or laboratory results or with chronic diseases or cardiovascular disorders. Lead blood levels were determined using air-acetylene flame atomic absorption spectrophotometry. The detectable limit was $1.23 \mu \mathrm{g} / \mathrm{dL}$. After the analyses of lead in blood, the following values were determined: minimum value, first quartile, median, third quartile, and maximum value; geometric mean; $95 \%$ confidence interval; experimental interval; and reference value.

Results. The reference values for lead in blood ranged from $1.20 \mu \mathrm{g} / \mathrm{dL}$ to $13.72 \mu \mathrm{g} /$ dL. The geometric mean was $5.5 \mu \mathrm{g} / \mathrm{dL}$.

Conclusions. In general, the values found in this study are lower than those that have been reported for other countries. Additional data should be gathered from Brazilian populations living in more-industrialized areas.

It is because of the need for the study of general populations in epidemiologic studies that community health agencies are particularly fitted for this type of investigation. Other health facilities are primarily concerned with persons already ill, and, although such populations may be used for certain types of retrospective studies, they cannot be used to determine the relative risk of developing a disease at a time when preventive measures could be instituted with a reasonable hope of influencing development or progression of the disease.

[La necesidad de trabajar con poblaciones generales en los estudios epidemio-lógicos hace que las agencias de salud comunitarias sean particularmente idóneas para este tipo de investigación. Otras instituciones de salud se dedican principalmente a tratar a personas ya enfermas y, aunque tales poblaciones pueden ser útiles para ciertos tipos de estudios retrospectivos, no lo son para determinar el riesgo relativo de desarrollar una enfermedad en un momento en que aún se podrían aplicar medidas preventivas con probabilidades de influir en el desarrollo o progresión de la enfermedad.]

Thomas R. Dawber, William B. Kannel, Lorna P. Lyell "An approach to longitudinal studies in a community: the Framingham study" Annals New York Academy of Sciences 1993;107:539-556 